\title{
Small steps towards Grand Unification \\ and the electron/positron excesses in cosmic-ray experiments
}

\author{
Masahiro Ibe $^{1}$ \\ ${ }^{1}$ SLAC National Accelerator Laboratory, Menlo Park, CA 94025
}

(Dated: June 25, 2009)

\begin{abstract}
We consider a small extension of the standard model by adding two Majorana fermions; those are adjoint representations of the $S U(2)_{L}$ and $S U(3)_{c}$ gauge groups of the standard model. In this extension, the gauge coupling unification at an energy scale higher than $10^{15} \mathrm{GeV}$ is realized when the masses of the triplet and the octet fermions are smaller than $10^{4} \mathrm{GeV}$ and $10^{12} \mathrm{GeV}$, respectively. We also show that an appropriate symmetry ensures a long lifetime of the neutral component of the triplet fermion whose thermal relic density naturally explains the observed dark matter density. The electron/positron excesses observed in recent cosmic-ray experiments can be also explained by the decay of the triplet fermion.
\end{abstract}

\section{INTRODUCTION}

The most beautiful framework for physics beyond the standard model is the grand unified theory (GUT) where the three gauge groups of the model are unified into a larger gauge group at a very high energy scale [1]. The most important prediction of the grand unified theory is the unification of the gauge coupling constants at a very high energy scale. Remarkably, the extrapolations of the three gauge coupling constants of the standard model to higher energies roughly suggest the unification of the gauge coupling constants.

Despite such a suggestion of the coupling unification, however, the precise measurements of the gauge coupling constants have revealed that three couplings do not coincide on one scale 2]. Another important prediction of the grand unified theory is the finite lifetime of the proton which decays via the interactions mediated by the heavy gauge bosons of the grand unified gauge theory. Unfortunately, the predicted lifetime, $\tau\left(p \rightarrow \pi^{0} e^{+}\right) \sim 10^{30} \mathrm{yr}$, is much shorter than the current experimental limit, $\tau\left(p \rightarrow \pi^{0} e^{+}\right)>8.2 \times 10^{33} \mathrm{yr}[\underline{3}] .^{1}$

The above lessons tell us that the grand unified theory requires additional particles below the unification scale, so that the three gauge couplings better agree with each other on a high energy scale and the unification scale is high enough to suppress the rate of the proton decay. One of the most successful extension of the standard model which satisfies those requirements is the supersymmetric standard model where superpartners for all the standard model particles have masses of order of the electroweak scale [4]. There, the unification is realized very precisely and the unification scale is raised to around $10^{16} \mathrm{GeV}$, which predicts a much longer lifetime of the proton than the current experimental limit.

In this paper, we consider a much smaller extension of the standard model which realizes the better unification and the higher unification scale than those in the standard model. Concretely, we just add two Majorana fermions; those are adjoint representations of the $S U(2)_{L}$ and $S U(3)_{c}$ gauge groups of the standard model. We name them "wino-like $(\tilde{w})$ " fermion and "gluino-like $(\tilde{g})$ " fermion, respectively, after the fashion of the supersymmetric standard model. The better unification and the higher unification scale are realized when the masses of the adjoint fermions satisfy $M_{\tilde{w}} \lesssim 10^{4} \mathrm{GeV}$ and $M_{\tilde{g}} \lesssim 10^{12} \mathrm{GeV}$ (see Ref. [5] for an earlier discussion on the effects of the adjoint fermions to the gauge coupling unification).

We go one step further. The scale of the mass of the gluino-like fermion, $M_{\tilde{g}} \lesssim 10^{12} \mathrm{GeV}$, is tempting to interrelate the mass to the breaking scale of the so-called Peccei-Quinn symmetry which is introduced to solve the strong CPproblem [ $[6]$. As we will see, the mass hierarchy between the two adjoint fermions can be explained with an appropriate choice of the charges of the fermions under the Peccei-Quinn symmetry.

As an interesting bonus of the introduction of the Peccei-Quinn symmetry, the interactions between the winolike fermion and the fermions in the standard model can be suppressed. As a result of the suppression, the neutral component of the wino-like fermion has a very long lifetime and is a candidate of the dark matter. In fact, the thermal relic density of the neutral wino-like fermion with a mass around $3 \mathrm{TeV}$ naturally explains the observed dark matter density, $\Omega_{\mathrm{DM}} h^{2}=0.1358_{-0.0036}^{+0.0037}$. 7 .

\footnotetext{
${ }^{1}$ Here, we are assuming the minimal gauge group of the grand unification, $S U(5)$, where the leptons and quarks are classified into the $\overline{\mathbf{5}}$ and $10[1]$.
} 

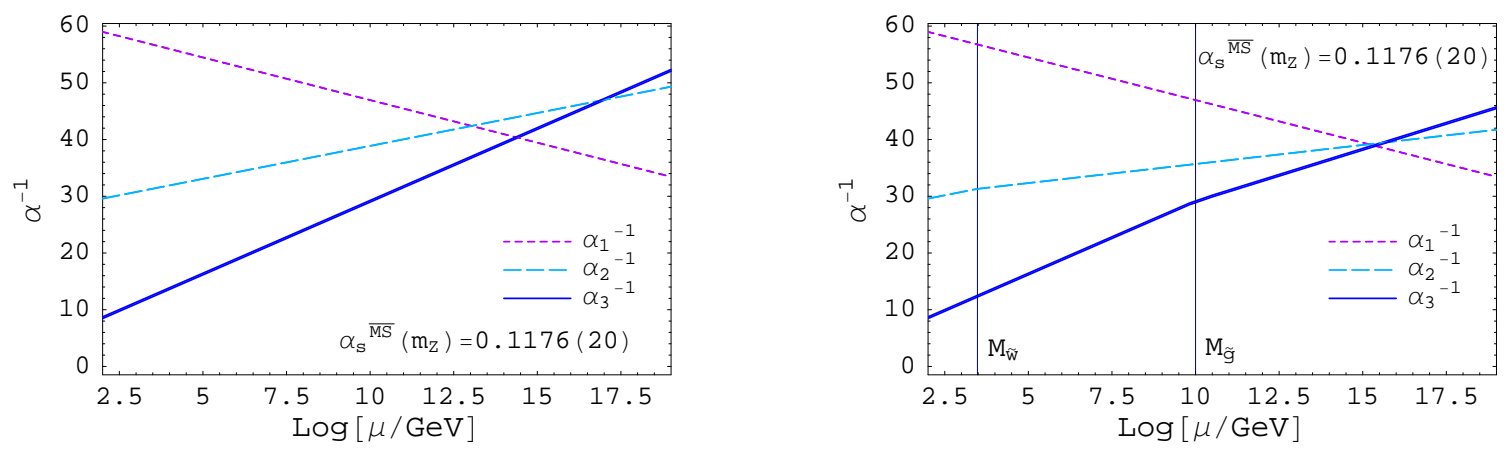

FIG. 1: The one-loop renormalization group evolutions of the gauge coupling constants in terms of $\alpha_{a}^{-1}$ in the standard model (left) and in the extended model (right). In the extended model, the masses of the wino-like and gluino-like fermions are taken to be $M_{\tilde{w}}=3 \mathrm{TeV}$ and $M_{\tilde{g}}=10^{10} \mathrm{GeV}$, respectively. In the figures, we use $\alpha_{3}\left(m_{Z}\right)^{\overline{\mathrm{MS}}}=0.1176(20), N_{H}=1$. We have also taken $m_{h}=117 \mathrm{GeV}$, and $m_{\text {top }}=171.3 \mathrm{GeV}$, although the results do not depend on these parameters significantly.

As another bonus, the observed electron/positron excesses at the PAMELA [8] and Fermi [9] experiments are also explained by the decay of the dark matter. As we will show, an appropriate charge assignment of the Peccei-Quinn symmetry leads to a lifetime of the dark matter which is suitable to explain the electron/positron excesses in cosmic ray.

The organization of the paper is as follows. In section [1 we show that the better unification and the higher unification scale are realized when the masses of the adjoint fermions, $M_{\tilde{w}}$ and $M_{\tilde{g}}$, satisfy $M_{\tilde{w}} \lesssim 10^{4} \mathrm{GeV}$ and $M_{\tilde{g}} \lesssim 10^{12} \mathrm{GeV}$. In section[II], we consider a $U(1)$ symmetry to address the origin of the mass hierarchy between the two adjoint fermions. There, we show that the $U(1)$ symmetry can be identified with the Peccei-Quinn symmetry. In sectionIV we show that the neutral component of the wino-like fermion has a very long lifetime with an appropriate choice of the Peccei-Quinn charge assignment. In section $\mathbb{V}$, we demonstrate how well the excesses of the electron/positron fluxes observed at the PAMELA and Fermi experiments can be explained by the decay of the wino-like fermion. The final section is devoted to conclusions.

\section{STEP 1: COUPLING UNIFICATION AND MASSES OF ADJOINT FERMIONS}

Let us begin with a basic test of the grand unification in an extension of the standard model with additional adjoint fermions; the test on how well the three gauge coupling constants unify at a high energy scale. Throughout this paper, we assume the minimal gauge group of the grand unification, $S U(5)$, where the leptons and quarks are classified into the $\overline{\mathbf{5}}$ and $\mathbf{1 0}$ representations [1]. The unification scale is estimated by using the one-loop renormalization group equations of the gauge coupling constants,

$$
\frac{d \alpha_{a}^{-1}}{d \ln \mu}=-\frac{b_{a}}{2 \pi}(a=1,2,3)
$$

where $\mu$ is the scale of the renormalization and the quantities $\alpha_{i}$ are related to the gauge coupling constants of the standard model gauge interactions by $\alpha_{i}=g_{i}^{2} / 4 \pi$. Here, $g_{1}$ is a rescaled gauge coupling of the $U(1)_{Y}$ gauge interaction, i.e. $g_{1}=\sqrt{5 / 3} g^{\prime}$. Above the electroweak scale, the coefficients of the beta functions are given by

$$
\begin{array}{llll}
b_{1}=4+\frac{N_{H}}{10}, & & \\
b_{2}=\frac{10}{3}-\frac{N_{H}}{6} & \left(\mu<M_{\tilde{w}}\right), & 2-\frac{N_{H}}{6} & \left(\mu>M_{\tilde{w}}\right), \\
b_{3}=-7 & \left(\mu<M_{\tilde{g}}\right), & -5 & \left(\mu>M_{\tilde{g}}\right),
\end{array}
$$

where $N_{H}$ is the number of Higgs doublets and $M_{\tilde{w}, \tilde{g}}$ denote the Majorana masses of the adjoint fermions.

In Fig. 1, we compare the renormalization group evolutions of $\alpha_{a}^{-1}$ in the standard model with those in the extended model at the one-loop level. In the extended model, we have taken $N_{H}=1, M_{\tilde{w}}=3 \mathrm{TeV}$ and $M_{\tilde{g}}=10^{10} \mathrm{GeV}$ as an example. Contrary to the standard model, the gauge coupling constants in the extended model coincide on one scale around $10^{15.5} \mathrm{GeV}$.

We quantify the degree of unification of the gauge coupling constants. For that purpose, let us remind ourselves that there can be sizable threshold corrections to the gauge coupling constants around the unification scale. Therefore, the 

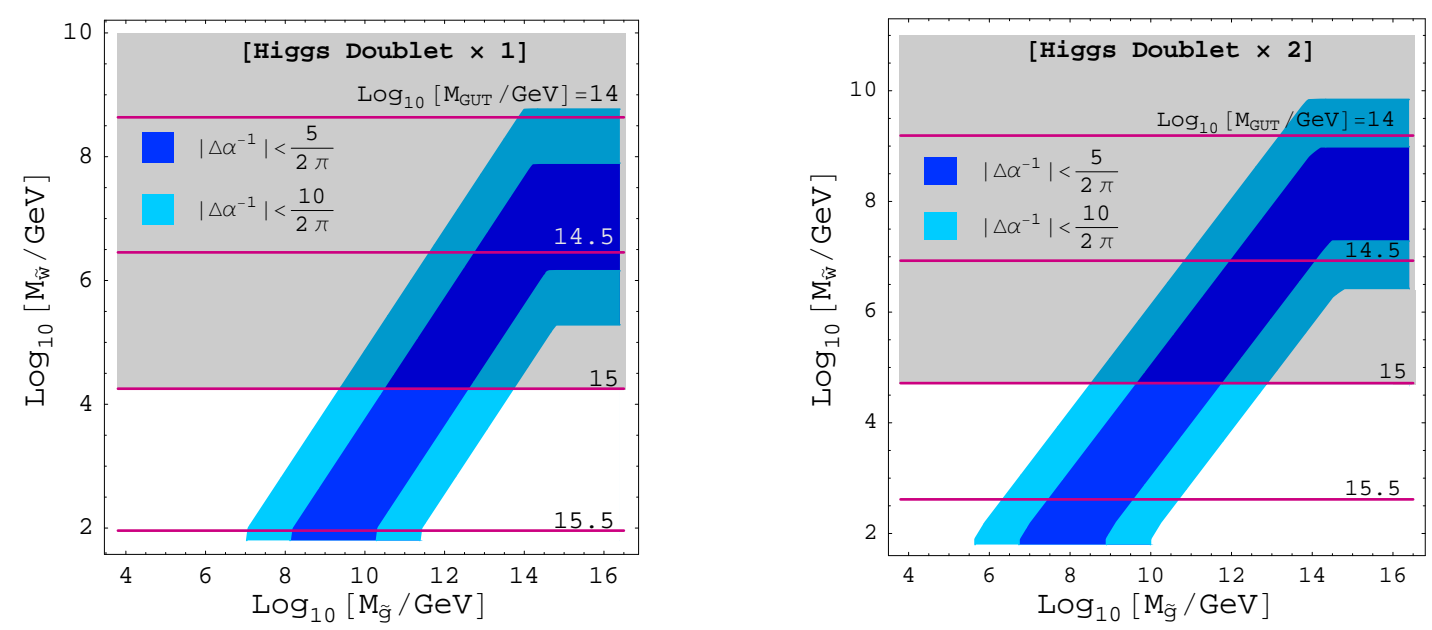

FIG. 2: The adjoint fermion masses which satisfy the unification test, i.e. $\left|N_{\mathrm{th}}\right|<5,10$ (see Eq. (3)), for one higgs doublet (left) and for two higgs doublets (right). The (light-)blue shaded regions correspond to the masses which satisfy $\left|N_{\mathrm{th}}\right|<5(10)$. For $M_{\tilde{g}} \gtrsim 10^{15} \mathrm{GeV}$, the degree of unification does not depend on $M_{\tilde{g}}$ since $M_{\tilde{g}}$ is higher than the unification scale $M_{\mathrm{GUT}}$ in this region. The horizontal lines show the contours of the unification scale. The gray-shaded region corresponds to $M_{\mathrm{GUT}}<10^{15} \mathrm{GeV}$ which is roughly excluded by the current lower limit on the proton lifetime (see discussion around Eq. (10)). In the figures, we have used $\alpha_{3}\left(m_{Z}\right)^{\overline{\mathrm{MS}}}=0.1176, m_{h}=117 \mathrm{GeV}$, and $m_{\text {top }}=171.3 \mathrm{GeV}$, although the results do not depend on those parameters significantly. In the case of the two higgs doublet model, we assumed that the threshold corrections at the electroweak scale is not so different from those in the one higgs doublet model. This assumption is also good enough for our purpose as long as the masses of the second higgs bosons are in the electroweak scale.

exact unification of the extrapolated gauge coupling constants does not have significant meaning, and there remains some freedom in how we define the unification, which depends on explicit models of the grand unified theory. In this study, instead of specifying models of the grand unified theory, we quantify the degree of unification in terms of the size of the required threshold correction at the unification scale, by defining the unification scale $M_{\mathrm{GUT}}$ and the threshold parameter $N_{\text {th }}$ by,

$$
\alpha_{1}\left(M_{\mathrm{GUT}}\right)=\alpha_{2}\left(M_{\mathrm{GUT}}\right) \equiv \alpha_{\mathrm{GUT}}, \quad \Delta \alpha^{-1}=\alpha_{\mathrm{GUT}}^{-1}-\alpha_{3}^{-1}\left(M_{\mathrm{GUT}}\right) \equiv \frac{N_{\mathrm{th}}}{2 \pi} .
$$

The parameter $N_{\text {th }}$ quantifies how large a threshold correction at the unification scale is required to realize a unified theory, and roughly speaking, it corresponds to the signed number of the charged particles (in the unit of the fundamental representation) which contribute to the threshold correction around the unification scale. For example, in the case of the supersymmetric standard model where masses of all the superparticles are of order of the electroweak scale, the threshold parameter satisfies $\left|N_{\mathrm{th}}\right| \lesssim 5[10] .^{2}$

In Fig. 2, we show the degree of unification in the $M_{\tilde{g}}-M_{\tilde{w}}$ plane for $N_{H}=1,2$. The figures show that the precise unification, $N_{\text {th }} \lesssim 5$, is realized for

$$
M_{\tilde{w}} \simeq 10^{-(6-8)} \times M_{\tilde{g}}, \quad \text { or } \quad M_{\tilde{w}} \simeq 10^{6-8} \mathrm{GeV} \quad \text { for } \quad M_{\tilde{g}} \gtrsim 10^{15} \mathrm{GeV},
$$

for $N_{H}=1$, and for

$$
M_{\tilde{w}} \simeq 10^{-(5-7)} \times M_{\tilde{g}}, \quad \text { or } \quad M_{\tilde{w}} \simeq 10^{7-9} \mathrm{GeV} \quad \text { for } \quad M_{\tilde{g}} \gtrsim 10^{15} \mathrm{GeV},
$$

for $N_{H}=2$.

Next, we consider the second test, the lifetime of the proton. In the minimal grand unified theory with the $S U(5)$ gauge group, the protons decay into pairs of the pion and the electron via the effective four fermi interactions (see for example Ref. [11]),

$$
\mathcal{L}=\frac{g_{\mathrm{GUT}}^{2}}{M_{V}^{2}}\left[A_{R}\left(\bar{d}_{R}^{\dagger} \bar{u}_{R}^{\dagger}\right)\left(u_{L} e_{L}\right)+A_{L}\left(1+\left|V_{u d}\right|^{2}\right)\left(u_{L} d_{L}\right)\left(\bar{u}_{R}^{\dagger} \bar{e}_{R}^{\dagger}\right)+\text { h.c. }\right]
$$

\footnotetext{
2 The parameter $N_{\mathrm{th}}$ is related to the threshold parameter $\varepsilon_{g}$ in Ref. [10] by $\varepsilon_{g}=N_{\mathrm{th}} / 4 \pi \times \alpha_{\mathrm{GUT}}$.
} 
which are mediated by the exchanges of the heavy gauge bosons of the grand unified theory. Here, $g_{\text {GUT }}$ is the unified gauge coupling constant, $g_{\mathrm{GUT}}^{2} / 4 \pi \simeq 1 / 40, M_{V}$ the mass of the heavy gauge bosons, $V_{u d} \simeq 0.974$ the $u d$-component of the Cabibbo-Kobayashi-Masukawa matrix. The coefficients $A_{R, L}$ represent the renormalization factors of the above operators from the unification scale to the lower energy scales. At the renormalization scale $\mu=2 \mathrm{GeV}$, the coefficients $A_{R, L}$ are given by,

$$
\begin{aligned}
A_{R, L} & =A_{R, L}^{\mathrm{SM}} \times\left(\frac{\alpha_{2}\left(M_{\tilde{w}}\right)}{\alpha_{\mathrm{GUT}}}\right)^{\frac{27}{12}\left(b_{2}^{-1}\left(\mu>M_{\tilde{w}}\right)-b_{2}^{-1}\left(\mu<M_{\tilde{w}}\right)\right)} \times\left(\frac{\alpha_{3}\left(M_{\tilde{g}}\right)}{\alpha_{\mathrm{GUT}}}\right)^{2\left(b_{3}^{-1}\left(\mu>M_{\tilde{g}}\right)-b_{3}^{-1}\left(\mu<M_{\tilde{g}}\right)\right)} \\
& \simeq A_{R, L}^{\mathrm{SM}} \times(1.0-1.2)
\end{aligned}
$$

for wide ranges of $M_{\tilde{w}}$ and $M_{\tilde{g}}$. The renormalization factors in the standard model, $A_{R, L}^{\mathrm{SM}}$, are given by $A_{R}^{\mathrm{SM}} \simeq 3$ and $A_{L}^{\mathrm{SM}} \simeq 3.2$ at $\mu=2 \mathrm{GeV}[11] .{ }^{3}$ From the above operators, the lifetime of the proton is given by,

$$
\tau\left(p \rightarrow \pi^{0} e^{+}\right) \simeq 1.4 \times 10^{34} \mathrm{yr} \times\left(\frac{A_{L, R}^{\mathrm{SM}}}{A_{L, R}}\right)^{2}\left(\frac{1 / 40}{\alpha_{G}}\right)^{2}\left(\frac{M_{V}}{10^{15.5} \mathrm{GeV}}\right)^{4}\left(\frac{0.06 \mathrm{GeV}^{2}}{\left|W_{0}\right|}\right)^{2}
$$

where $W_{0}=-0.06 \pm 0.018 \mathrm{GeV}^{2}$ is the form factor of the proton decay operators between the proton and the pion states calculated with lattice QCD [12]. ${ }^{4}$

By comparing the predicted lifetime with the current experimental limit, $\tau\left(p \rightarrow \pi^{0} e^{+}\right)>8.2 \times 10^{33}$ yr $[3]$, we obtain a lower limit on the heavy gauge boson mass,

$$
M_{V} \gtrsim 10^{15.4} \mathrm{GeV} \times\left(\frac{A_{L, R}}{A_{L, R}^{\mathrm{SM}}}\right)^{1 / 2}\left(\frac{\alpha_{G}}{1 / 40}\right)^{1 / 2}\left(\frac{\left|W_{0}\right|}{0.06 \mathrm{GeV}^{2}}\right)^{1 / 2} .
$$

Then, by expecting that the mass of the heavy gauge bosons is not so far from the unification scale, we can translate the above lower limit to a limit on the unification scale. Notice that the exact relation between the unification scale and the gauge boson mass depends on models of the grand unified theory. In this study, instead of specifying models of the unified theory, we just assume that the gauge boson mass is of order of the unification scale and we put a rough lower limit on the unification scale,

$$
M_{\mathrm{GUT}} \gtrsim 10^{15} \mathrm{GeV}
$$

In Fig.2 2 the shaded regions satisfy the test of the proton lifetime; $M_{\mathrm{GUT}} \gtrsim 10^{15} \mathrm{GeV}$. The figures show that the regions of relatively heavy wino-like fermion are excluded by the second test. As a result, the masses of the adjoint fermions which pass both the tests are as follows;

$$
M_{\tilde{w}} \simeq 10^{-(6-8)} \times M_{\tilde{g}} \quad\left(M_{\tilde{w}} \lesssim 10^{4} \mathrm{GeV}\right),
$$

for $N_{H}=1$ and

$$
M_{\tilde{w}} \simeq 10^{-(5-7)} \times M_{\tilde{g}}\left(M_{\tilde{w}} \lesssim 10^{4.5} \mathrm{GeV}\right),
$$

for $N_{H}=2$.

\section{STEP 2: ORIGIN OF MASSES OF ADJOINT FERMIONS}

In the previous section, we have shown that the small extension of the standard model with adjoint fermions predicts better unification with a longer lifetime of the proton than those in the standard model for

$$
M_{\tilde{w}} \lesssim 10^{4} \mathrm{GeV}, \quad M_{\tilde{g}} \lesssim 10^{12} \mathrm{GeV}
$$

\footnotetext{
3 The renormalization factors are slightly smaller than those in Ref. [11] due mainly to the use of the different standard model parameters.

4 The proton decay rate with $W_{0}=-0.06 \mathrm{GeV}^{2}$ corresponds to that expressed in terms of the form factor with the proton and the vacuum, $\alpha_{H}=0.005 \mathrm{GeV}^{3}$, which is often used to represent the proton lifetime in the literature. In the chiral perturbation theory, those parameters are related by $W_{0}=\alpha_{H}\left(1+g_{A}\right) / \sqrt{2} f_{\pi}$, with the tree-level pion decay constant $f_{\pi} \simeq 131 \mathrm{MeV}$ and the nucleon axial charge, $g_{A} \simeq 1.22$. See Ref. [12] for detailed discussions on the lattice simulations on those form factors.
} 
In this section, we try to explain the mass spectrum of the adjoint fermions by considering a spontaneous symmetry breaking of a global $U(1)$ symmetry. As we will see, the $U(1)$ symmetry can be identified with the Peccei-Quinn symmetry [6], and hence, the strong CP-problem is solved automatically.

Let us first assume that the model is invariant under global $U(1)$ chiral rotations,

$$
\tilde{g} \rightarrow \tilde{g}^{\prime}=e^{i \alpha / 2} \tilde{g}, \quad \tilde{w} \rightarrow \tilde{w}^{\prime}=e^{i \alpha} \tilde{w},
$$

with an angle $\alpha .{ }^{5}$ Under this symmetry, the masses of the adjoint fermions are forbidden. Next let us further assume that the chiral symmetry is broken spontaneously at around $10^{8-12} \mathrm{GeV}$ by a condensation of a scalar field $X$ which rotates under the above chiral symmetry by,

$$
X \rightarrow X^{\prime}=e^{-i \alpha} X \quad\left(\langle X\rangle \simeq 10^{8-12} \mathrm{GeV}\right) .
$$

With this spontaneous breaking, the gluino-like fermion obtains a mass from a direct coupling with $X$,

$$
\mathcal{L}_{\tilde{g}} \simeq \frac{1}{2} X \tilde{g} \tilde{g}+h . c .
$$

which results in $M_{\tilde{g}} \simeq\langle X\rangle \simeq 10^{8-12} \mathrm{GeV}$. Here, we have neglected coefficients of the order one.

The mass term of the wino-like fermion, on the other hand, is still suppressed by the chiral symmetry, and it begins with a dimension five operator suppressed by $M_{\mathrm{GUT}}$,

$$
\mathcal{L}_{\tilde{w}} \simeq \frac{1}{2} \frac{X^{2}}{M_{\mathrm{GUT}}} \tilde{w} \tilde{w}+\text { h.c. }
$$

when we assume that the interactions between the scalar $X$ and the wino-like fermion are mediated by fields of masses of order of the unification scale. Once the dimension five operator is generated at the unification scale, the mass of the wino-like fermion is given by,

$$
M_{\tilde{w}} \simeq \frac{\langle X\rangle^{2}}{M_{\mathrm{GUT}}} \simeq 3 \mathrm{TeV} \times\left(\frac{\langle X\rangle}{10^{9.5} \mathrm{GeV}}\right)^{2}\left(\frac{10^{15.5} \mathrm{GeV}}{M_{\mathrm{GUT}}}\right)
$$

which is consistent with the unification tests in the previous section. As a result, we found that the masses of the adjoint fermions can be naturally explained by spontaneous breaking of a chiral symmetry at the intermediate scale.

The interesting outcome of the above chiral symmetry is that the chiral symmetry plays the role of the so-called Peccei-Quinn symmetry [13, 14]. That is, the above chiral rotation is anomalous to the $S U(3)_{c}$ gauge symmetry, and the axion resulting from the spontaneous breaking of the chiral symmetry cancels the $\theta$ angle in QCD which is otherwise required to be tuned to a very small value, $|\theta| \lesssim 10^{-10}[15]$. It should be noted that the properties of the axion are consistent with the astrophysical and the cosmological constraints for $\langle X\rangle \simeq 10^{9-12} \mathrm{GeV}$ (see for example Ref. [16] and references there in for detailed discussion on astrophysical constraints on the Peccei-Quinn breaking scale). Therefore, the above small extension of the standard model which satisfies the unification can be naturally integrated with the solution to the strong $\mathrm{CP}$-problem.

\section{STEP 3: STABILITY OF ADJOINT FERMIONS AND DARK MATTER DENSITY}

Recent observations of the electron/positron excesses in the PAMELA [8] and Fermi [9] experiments strongly suggest the existence of a new source of electron/positron fluxes. The most interesting candidate of the new source which is related to physics beyond the standard model is the decay of the dark matter with a mass in the TeV range. Therefore, it is an interesting question whether the above wino-like fermion can be a candidate of the dark matter, and on top of that, it explains the observed electron/psoitron excesses in cosmic ray. (The earlier works on the electron/positron excesses from the decay of the wino-like dark matter of a mass in the TeV range have been done in Refs. [17, 18] in a model independent way, and in Refs. [19, 20] in the context of the supersymmetric standard model.)

Before going to the stability of the wino-like fermion, it should be checked whether the neutral component of the triplet wino-like fermion is the lightest component. The dominant mass splitting between the neutral and the charged

\footnotetext{
${ }^{5}$ The above charge assignment suggests that the gluino-like and the wino-like fermions stem from different multiplets in the grand unified theory, although we do not pursue explicit models of the grand unified theory in this paper.
} 


\begin{tabular}{c|ccccccc} 
& $H$ & $\ell_{L}$ & $\bar{e}_{R}$ & $q_{L}$ & $\bar{u}_{R}$ & $\bar{d}_{R}$ & $\tilde{w}$ \\
\hline$S U(2)$ & $\mathbf{2}$ & $\mathbf{2}$ & $\mathbf{1}$ & $\mathbf{2}$ & $\mathbf{1}$ & $\mathbf{1}$ & $\mathbf{3}$ \\
$U(1)_{\mathrm{Y}}$ & $-1 / 2$ & $-1 / 2$ & 1 & $1 / 6$ & $-2 / 3$ & $1 / 3$ & 0 \\
$U(1)_{\mathrm{PQ}}$ & $12 / 5$ & $-18 / 5$ & $6 / 5$ & $6 / 5$ & $6 / 5$ & $-18 / 5$ & 1
\end{tabular}

TABLE I: The Peccei-Quinn-charges of the standard model fields. Here, we also show the charges under the standard model gauge group; $S U(2) \times U(1)_{Y}$. We assign the same charges to all the three generations of the standard model fermions.

components in the wino-like fermion comes from the one-loop weak gauge boson exchange diagrams [21], which is given by

$$
\begin{aligned}
\Delta M_{\tilde{w}} & =M_{\tilde{w}^{ \pm}}-M_{\tilde{w}^{0}}=\frac{g_{2}^{2}}{16 \pi^{2}} M_{\tilde{w}}\left[f\left(r_{W}\right)-\cos ^{2} \theta_{W} f\left(r_{Z}\right)-\sin ^{2} \theta_{W} f(0)\right], \\
& \simeq 161 \mathrm{MeV}-165 \mathrm{MeV} \quad\left(\text { for } M_{\tilde{w}}=1 \mathrm{TeV}-10 \mathrm{TeV}\right),
\end{aligned}
$$

where $f(r)=\int_{0}^{1} d x\left(2+2 x^{2}\right) \ln \left[x^{2}+(1-x) r^{2}\right], r_{i}$ denotes the weak gauge boson masses normalized by the mass of wino-like fermion, $r_{i}=m_{i} / M_{\tilde{w}}$. Here, we have assumed that the direct interactions between the wino-like fermion and the standard model fields are suppressed, which will be justified in the following discussion. As a result, we see that the neutral component is the lightest component of the wino like fermion. This splitting allows the charged components decay into the neutral component and a virtual $W^{ \pm}$bosons which end up with $\pi^{ \pm}$or lepton pairs. These are crucial features of the wino-like fermion as a dark matter candidate, otherwise the wino-like fermion leads to a charged dark matter.

Now, let us ask whether the neutral component is stable or not. The easiest way to achieve the stability is to introduce a $Z_{2}$ symmetry under which the wino-like fermion changes the sign. With the $Z_{2}$ symmetry, we can forbid any interactions which cause the decay of the neutral component of the wino-like fermion. It is, however, more attractive if the stability of the neutral component is ensured by symmetries which are introduced for some other reasons than the the stability of the dark matter. In the followings, we show that an appropriate charge assignment of the Peccei-Quinn symmetry to the standard model fields leads to a stability of the wino-like fermion.

The lowest dimensional interactions which cause the decay of the wino-like fermion are given by,

$$
\mathcal{L}_{\text {decay }}=c_{i} H_{a}^{*} \tilde{w}^{A} t_{b}^{A a} \ell_{L i}^{b},
$$

where $c_{i}$ denotes a coefficient, $t^{A}$ the generators of $S U(2), H$ the higgs doublet, and $\ell_{L i}$ the lepton doublets of the flavor indices $i=1,2,3$. In the followings, we assume $N_{H}=1$, although we can extend our discussion for $N_{H}=2$, straightforwardly. With the above operators, the decay rate of the wino-like fermion is given by,

$$
\Gamma_{\tilde{w}}\left(\tilde{w}^{0} \rightarrow \ell^{ \pm} W^{\mp}, \nu Z, \nu h\right) \simeq \frac{c_{i}^{2}}{2 \pi} M_{\tilde{w}} .
$$

Here, we have summed all the possible final states. Therefore, in order for the wino-like fermion to be a dark matter candidate, $c_{i}$ must be highly suppressed.

To suppress the above operators, we give charges to the standard model fields under the Peccei-Quinn symmetry. In Table. I. we give an example of the charge assignment which suppresses the operators in Eq. (20). With this charge assignment, the operators in Eq. (20) have the corresponding Peccei-Quinn charge -5, and hence, they are highly suppressed. That is, when the interactions between the scalar $X$ and the standard model fields as well as the wino-like fermions are mediated by the fields of masses of order of the unification scale, the above operators only come from the effective operators,

$$
\mathcal{L}_{\text {decay }} \simeq \frac{X^{* 5}}{M_{\mathrm{GUT}}^{5}} H_{a}^{*} \tilde{w}^{A} t_{b}^{A a} L_{i}^{b}
$$

and hence, the coefficient $c_{i}$ is highly suppressed by $\left(\langle X\rangle / M_{\mathrm{GUT}}\right)^{5}$. As a result of the suppression, the wino-like fermions decay and the lifetime of the neutral wino-like fermion is given by,

$$
\tau_{\tilde{w}} \simeq 4 \times 10^{25} \sec \times\left(\frac{3 \mathrm{TeV}}{M_{\tilde{w}}}\right)\left(\frac{10^{9.5} \mathrm{GeV}}{\langle X\rangle}\right)^{10}\left(\frac{M_{\mathrm{GUT}}}{10^{15.5} \mathrm{GeV}}\right)^{10} .
$$



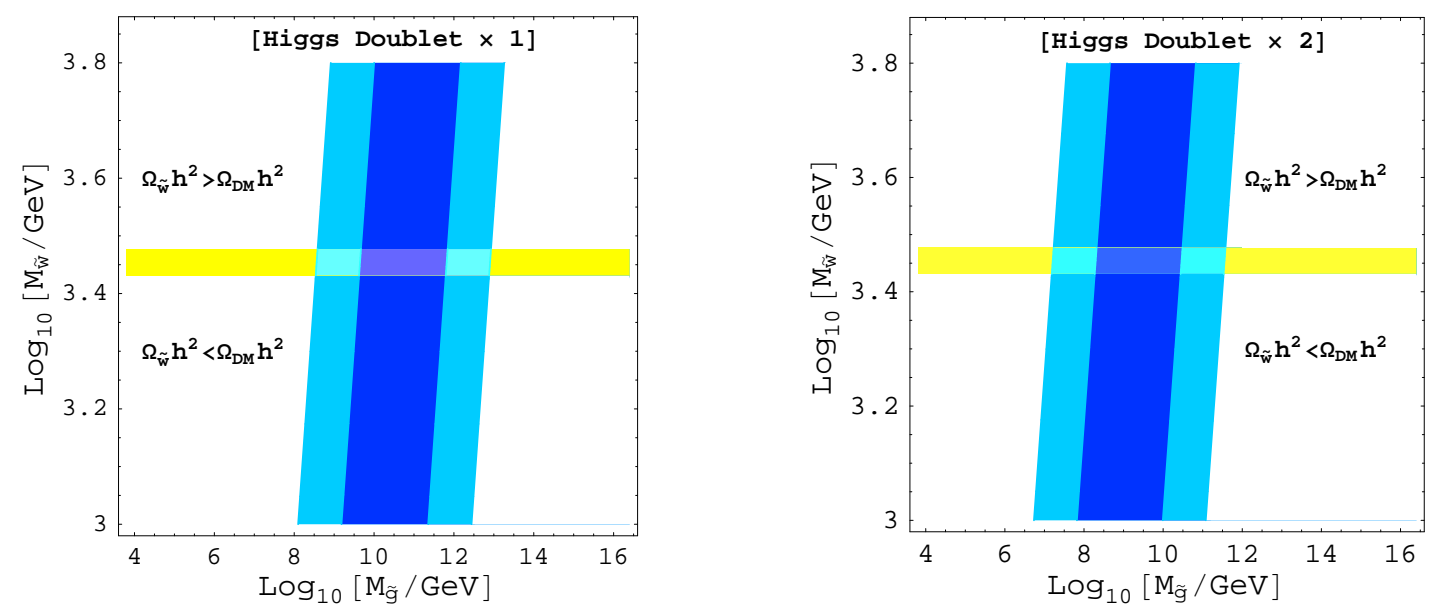

FIG. 3: The enlarged views of Fig.2 On the yellow bands, the thermal relic density is consistent with the observed dark matter density, which corresponds to $2.7 \mathrm{TeV} \lesssim M_{\tilde{w}} \lesssim 3 \mathrm{TeV}$. Above the bands, the thermal relic density is larger than the observed dark matter density, while it is smaller below the bands.

Therefore, the lifetime of the neutral wino-like fermion can be long enough to be a dark matter candidate. Moreover, the lifetime is in an appropriate range to explain the electron/positron excesses by the decay of the dark matter. ${ }^{6}$

We next consider the thermal relic abundance of the wino-like fermion. Since the interactions between the wino-like fermion and the matter fields of the standard model are highly suppressed, the dominant annihilation process is the one into two $W$-bosons via the $t$-channel exchange of the charged wino-like fermions. The perturbative analysis on this process gives the thermal relic density of the wino-like fermion [22],

$$
\Omega_{\tilde{w}} h^{2} \simeq 0.1 \times\left(\frac{M_{\tilde{w}}}{2 \mathrm{TeV}}\right)^{2} \quad(\text { perturbative }) .
$$

As pointed out in Ref. 23], however, the thermal relic abundance is significantly changed by a non-perturbative effect called the Sommerfeld enhancement when the neutral and the charged wino-like fermions are almost degenerate. The resultant mass range of the wino-like fermion which is consistent with the observed dark matter density is then given by [23],

$$
2.7 \mathrm{TeV} \lesssim M_{\tilde{w}} \lesssim 3.0 \mathrm{TeV} \quad \text { (non-perturbative) }
$$

In Fig.3, we show the corresponding parameter region on the $M_{\tilde{g}}-M_{\tilde{w}}$ plane. From the figure, we see that the masses which have passed the unification tests are consistent with the mass of the wino-like fermion which explain the observed dark matter density.

Before closing this section, we comment on the contributions of the standard model quarks to the $S U(3)_{c}$ anomaly of the Peccei-Quinn symmetry. As we see from the Table【, the contribution in the quark sector is cancelled, i.e.

$$
\frac{6}{5}(2+1)-\frac{18}{5}=0
$$

where the first term denotes the contribution from $q_{L}$ and $\bar{u}_{R}$, while the second term from $\bar{d}_{R}$. This cancellation can be understood by remembering that the above charge assignment can be expressed by,

$$
Q_{\mathrm{PQ}}^{\mathrm{SM}}=-\frac{24}{5} Q_{Y}-6 Q_{B-L},
$$

where both of $Q_{Y}$ and $Q_{B-L}$ are anomaly free. Therefore, the anomaly of the Peccei-Quinn symmetry to the $S U(3)_{c}$ gauge symmetry only comes from the gluino-like fermion under this charge assignment.

6 The other higher dimensional operators which also cause the decay of the neutral wino-like fermion are more suppressed by factors of the unification scale and by the symmetry. It should be also noted that when there are four fermion interactions such as $\tilde{w} \bar{e}_{R} \ell_{L i} \ell_{L j}$, the operators in Eq. (20) are induced radiatively and dominate the decay process. Thus, in the non-supersymmetric models, the decay modes via the four fermion interactions such as $\tilde{w} \rightarrow \ell+\ell+\nu$ cannot be the dominant mode. 


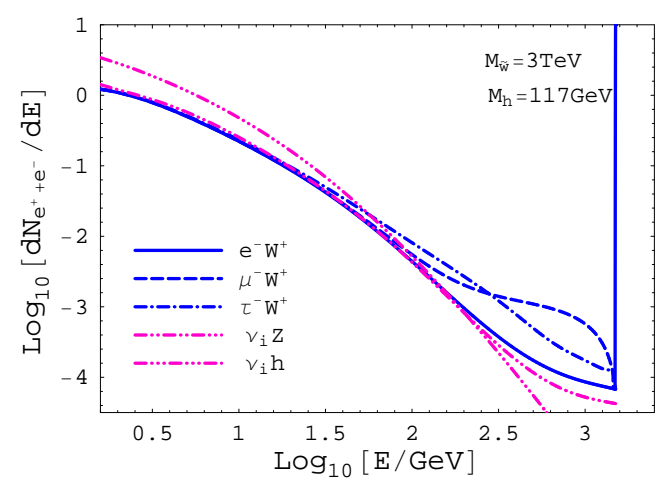

FIG. 4: The fragmentation functions into electrons and positrons of the each decay modes. We used the program PHYTIA [26] to obtain the functions. In this figure, we have taken $M_{\tilde{w}}=3 \mathrm{TeV}$ and $m_{h}=117 \mathrm{GeV}$.

\section{ELECTRON/POSITORON EXCESSES FROM DECAY OF DARK MATTER}

In the previous section, we showed that the wino-like fermion is a good candidate of the dark matter with the help of the Peccei-Quinn symmetry, and the thermal relic density explains the observed dark matter density for $M_{\tilde{w}} \simeq 3 \mathrm{TeV}$. Besides, with an appropriate choice of the Peccei-Quinn charges, the lifetime of the dark matter can be in an appropriate range for an explanation of the observed electron/positron excesses in cosmic ray, i.e. $\tau_{\tilde{w}} \sim 10^{26}$ sec. In fact, the decay mode of the wino-like fermion, $\tilde{w} \rightarrow \ell^{ \pm} W^{\mp}$ has been studied extensively in Ref. [17, 18], and the observed cosmic-ray spectra can be well fitted for $\ell=\mu$ with $M_{\tilde{w}}=3 \mathrm{TeV}$.

In this section, we apply their analysis to the wino-like fermion dark matter which decays via the operators in Eq. (21). According to the results in Refs. [17, 18], we concentrate on the case with $\left|c_{2}\right| \gg\left|c_{1,3}\right|$, so that the mode into $\ell=\mu$ is the dominant one. The important difference of our analysis from the generic analysis is that the decay mode $\tilde{w} \rightarrow \ell^{ \pm} W^{\mp}$ is accompanied by the other decay modes, $\tilde{w} \rightarrow \nu Z$ and $\tilde{w} \rightarrow \nu h$ with the branching ratios, ${ }^{7}$

$$
\operatorname{Br}\left(\tilde{w} \rightarrow \mu^{ \pm} W^{\mp}\right)=0.5, \quad \operatorname{Br}\left(\tilde{w} \rightarrow \nu_{2}^{(\dagger)} Z\right)=\operatorname{Br}\left(\tilde{w} \rightarrow \nu_{2}^{(\dagger)} h\right)=0.25 .
$$

In Fig.4, we show the fragmentation functions of the each decay modes into electrons and positions for $M_{\tilde{w}}=3 \mathrm{TeV}$. The figure shows that the contribution from the $Z$ and $h$ modes increase the number of the low energy electrons and positirons compared with those in the pure $W$ mode.

The predicted electron/positron spectrum in cosmic ray is shown in Fig. 囵 for the dark matter lifetime $\tau_{\tilde{w}}=10^{26}$ sec with the branching fractions given in Eq. (28) ${ }^{8}$ The analysis on the propagation of the electron/positron fluxes in the galaxy is based on that given in Ref. [27], and we used numerical approximated Green function with the choice of the MED propagation model in the reference. As for the background electron/positron spectra, we used the ones given in the same reference. The figure shows that the model fits the data of the Fermi experiment quite well. In the right panel of Fig. 5 , we also show the predicted positron fraction. In the figure, we have taken into account the solar modulation effect in the current solar cycle [31]. The figure also show that the positron fraction can be well fitted by the current model.

The weak gauge bosons and the higgs boson in the final states of the dark matter decays also fragment into protons/antiprotons. Such fragmentations into protons/antiprotons are severely constrained by the PAMELA experiment which shows no excess in the antiproton fraction [34]. ${ }^{9}$ In the left panel of Fig. 66 we show the fragmentation functions into the protons and antiprotons of $W^{ \pm}, Z$ and $h$ in the final states of the dark matter decay for $M_{\tilde{w}}=3 \mathrm{TeV}$. The figure shows that sizable numbers of the protons/antiprotons are expected from the fragmentations of those bosons. In

7 The above branching ratios are similar to the ones considered in the decaying gravitino dark matter scenarios 24, 25].

8 In Ref. [18], a similar spectrum is obtained for $\tau_{\tilde{w}}=2.1 \times 10^{26}$ sec. The difference of the chosen lifetimes reflects the difference of the branching ratio of the $\tilde{w} \rightarrow \mu^{ \pm} W^{\mp}$ mode, which requires the twice larger decay rate in our case.

9 This is a remarkable difference of the non-supersymmetric wino-like dark matter in comparison with the wino-like dark matter in the supersymmetric context. In the supersymmetric models such as Ref. [19], the decay of the wino-like dark matter proceeds via the dimension six operators $\tilde{w} \bar{e}_{R} \ell_{L} \ell_{L}$ which dominate over the dimension four operators $\tilde{w} \ell_{L} H^{*}$. On the other hand, in the case of the non-supersymmetric wino-like fermion, the later dimension four operators are generated radiatively from the former dimension six operators and the decay process is dominated by the dimension four operators. This difference may allow us to investigate whether the supersymmetry is behind the wino-like dark matter through the observation of the antiproton flux in cosmic ray. 

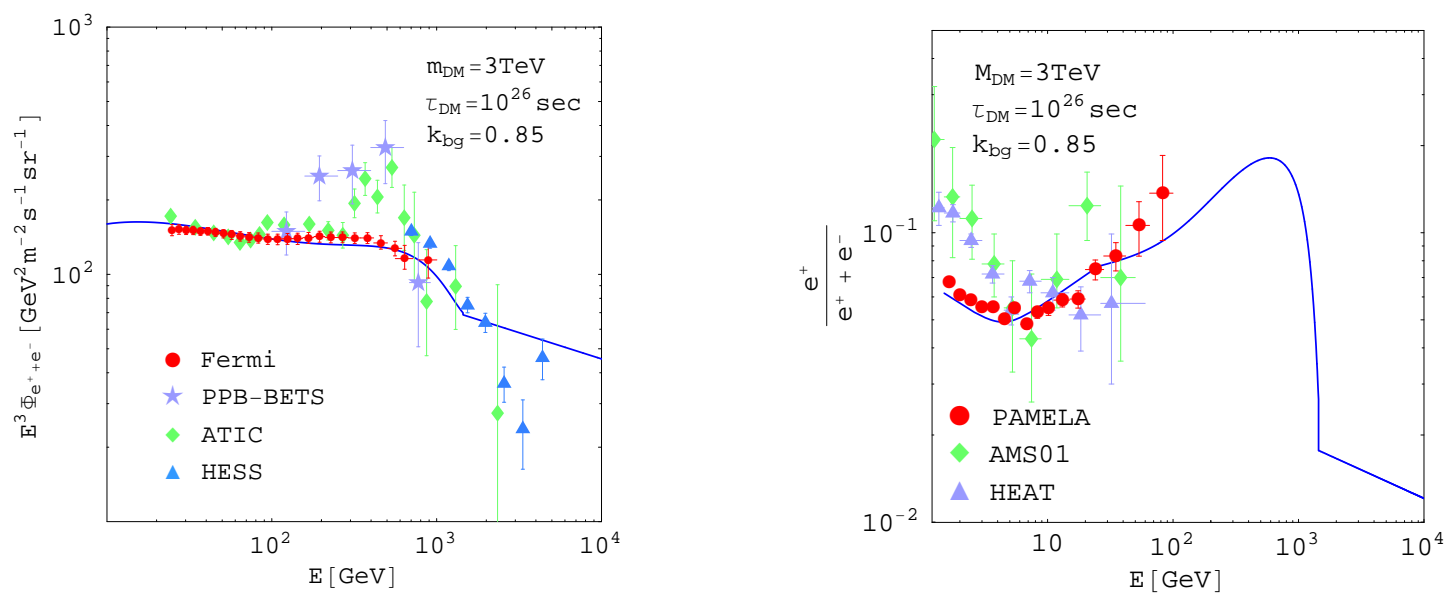

FIG. 5: Left) The predicted electron/positron flux in cosmic ray for the branching ratio in Eq. (28). The propagation of the electron/positron flux in the galaxy is analyzed based on a numerically approximated Green function given in Ref. [27] (the MED propagation model). The prediction is compared with the experimental data 9, 28, 29, 30]. Right) The predicted positron fraction in cosmic ray for the same dark matter decay modes with the experimental data [8, 32, 33].

the right panel of Fig. 6, we show the predicted antiproton fraction in cosmic ray for $\tau_{\tilde{w}}=10^{26}$ sec and the branching fraction in Eq. (28). In our analysis, we again used the numerical Green functions of the proton/antiproton propagation for three different diffusion models given in Ref. [27]. The background proton spectrum is borrowed from Ref. [35]. The figure shows that the predicted fraction is contradict with the observed fraction in some diffusion parameters. Thus, the decay mode in Eq. (28) with $\tau_{\tilde{w}}=10^{26} \mathrm{sec}$ for $M_{\tilde{w}}=3 \mathrm{TeV}$ is somewhat disfavored from the antiproton fraction observed in PAMELA experiments, although not completely excluded.

Before closing this section, we comment on the cosmic ray from the annihilation of the wino-like dark matter. As discussed in Ref. [36], the annihilation cross section of the dark matter in our galaxy is also enhanced by the Sommerfeld enhancement. The enhancement is, however, not so significant for $2.7 \mathrm{TeV} \lesssim M_{\tilde{w}} \lesssim 3 \mathrm{TeV}$, and the resultant cosmic ray from the annihilation are much smaller than that from the decay of the dark matter with a lifetime in the range of $10^{26} \mathrm{sec}$.

\section{CONCLUSIONS}

In this study, we found that the small extension of the standard model with adjoint fermions allows the better unification of the three gauge coupling constants of the standard model with a long enough proton lifetime, when the adjoint fermions have masses in ranges of $M_{\tilde{w}} \lesssim 10^{4} \mathrm{GeV}$ and $M_{\tilde{g}} \lesssim 10^{12} \mathrm{GeV}$. We also discussed that the neutral wino-like fermion can be a good candidate for the dark matter whose thermal relic density naturally explains the observed dark matter density. With an appropriate choice of the Peccei-Quinn charges, we also found that the lifetime of the neutral component of the wino-like fermion can be an appropriate range to explain the excesses of the electron/positron fluxes in cosmic ray in recent experiments.

It should be noted that the unification scale which is consistent with the dark matter density is not much higher than $10^{15} \mathrm{GeV}$, and hence, the masses of the heavy gauge bosons which mediate the proton decay are expected to be close to the current limit, $M_{V} \gtrsim 10^{15.4} \mathrm{GeV}$. Therefore, the model predicts rather short lifetime of the proton, $\tau_{p}=O\left(10^{34-35}\right)$ sec, which will hopefully be soon detected even at the current detectors such as Super-Kamiokande. This is a distinctive prediction in comparison with the supersymmetric standard model where the typical lifetime of the proton decaying via the gauge boson exchange is $O\left(10^{36}\right)$ sec. $^{10}$

The direct searches of the dark matter to detect the recoils of nuclei by the dark matter-neclei collision will give a clear evidence of the dark matter. As shown in Ref. [38], the cross-section of the direct detection of the wino-like

10 In some classes of the grand unified model in the supersymmetric model, the lifetime of the proton decaying via the gauge boson exchange is rather short [37]. 

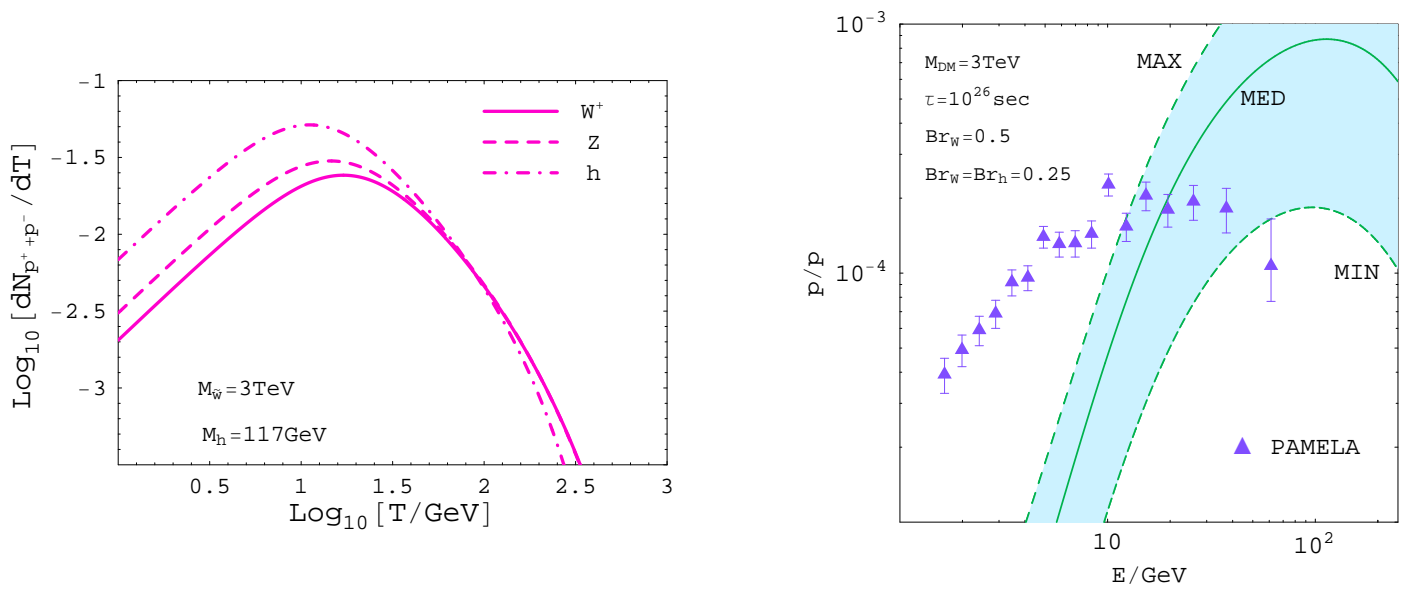

FIG. 6: Left) The fragmentation functions of $W, Z$ and $h$ into the protons and antiprotons. We used the program PHYTIA 26] to obtain the functions and assumed $m_{h}=117 \mathrm{GeV}$. Right) Predicted anti-proton and proton ratio in cosmic ray from the dark matter decay with the branching ratios in Eq. (28). The propagations of the proton/antiproton fluxes in the galaxy are analyzed based on a numerically approximated Green function given in Ref. 27] for three diffusion models, MAX, MED and MIN. We compare the prediction with the experimental date in Ref. 34]. The background proton spectrum is borrowed from Ref. [35].

dark matter is around $10^{-45} \mathrm{~cm}^{2}$ for $M_{\tilde{w}} \simeq 3 \mathrm{TeV}$, which is within reach of future experiments such as SuperCDMS experiment [39].

The detection of the wino-like fermion at the collider experiments is also interesting. As we have mentioned, the charged components of the wino-like fermion decay into a neutral wino-like fermion and the charged pion with the lifetime of $\mathcal{O}\left(10^{-10}\right)$ sec. Thus, once they are produced at collider experiments, they may leave displaced vertices which help us to detect the wino-like fermions at future experiments. ${ }^{11}$

Finally, we comment on the fate of the gluino-like fermion. Since it has a rather heavy mass, $M_{\tilde{g}} \simeq 10^{9-12} \mathrm{GeV}$, the cosmic abundance of the gluino-like fermion is highly suppressed as long as the temperature of the universe after inflation is much lower than $M_{\tilde{g}}$.

\section{Acknowledgements}

M. I. appreciate T.T Yanagida for stimulating discussions. M. I. also appreciate S. Shirai and F. Takahashi for a lot of advices in the analysis of cosmic ray spectra. The work of M. I. was supported by the U.S. Department of Energy under contract number DE-AC02-76SF00515.

[1] H. Georgi and S. L. Glashow, Phys. Rev. Lett. 32, 438 (1974); H. Georgi, H. R. Quinn and S. Weinberg, Phys. Rev. Lett. 33, 451 (1974); see also the review by, P. Langacker, Phys. Rept. 72, 185 (1981).

[2] J. R. Ellis, S. Kelley and D. V. Nanopoulos, Phys. Lett. B 260, 131 (1991). U. Amaldi, W. de Boer and H. Furstenau, Phys. Lett. B 260, 447 (1991); P. Langacker and M. x. Luo, Phys. Rev. D 44, 817 (1991); C. Giunti, C. W. Kim and U. W. Lee, Mod. Phys. Lett. A 6 (1991) 1745.

[3] H. Nishino et al. [Super-Kamiokande Collaboration], Phys. Rev. Lett. 102, 141801 (2009) [arXiv:0903.0676 [hep-ex]].

[4] S. Dimopoulos, S. Raby and F. Wilczek, Phys. Rev. D 24, 1681 (1981); S. Dimopoulos and H. Georgi, Nucl. Phys. B 193, 150 (1981); L. E. Ibanez and G. G. Ross, Phys. Lett. B 105, 439 (1981); N. Sakai, Z. Phys. C 11, 153 (1981); M. B. Einhorn and D. R. T. Jones, Nucl. Phys. B 196, 475 (1982); W. J. Marciano and G. Senjanovic, Phys. Rev. D 25, 3092 (1982).

[5] N. V. Krasnikov, Phys. Lett. B 306, 283 (1993).

[6] R. D. Peccei and H. R. Quinn, Phys. Rev. Lett. 38, 1440 (1977); R. D. Peccei and H. R. Quinn, Phys. Rev. D 16, 1791 (1977).

\footnotetext{
${ }^{11}$ See related works for the detection of the wino-like fermion in the lower mass region at the LHC experiments [40].
} 
[7] E. Komatsu et al. [WMAP Collaboration], Astrophys. J. Suppl. 180, 330 (2009) [arXiv:0803.0547 [astro-ph]].

[8] O. Adriani et al. [PAMELA Collaboration], Nature 458, 607 (2009) [arXiv:0810.4995 [astro-ph]].

[9] A. A. Abdo et al. [The Fermi LAT Collaboration], arXiv:0905.0025 [astro-ph.HE].

[10] J. Bagger, K. T. Matchev and D. Pierce, Phys. Lett. B 348, 443 (1995) [arXiv:hep-ph/9501277]; D. M. Pierce, J. A. Bagger, K. T. Matchev and R. j. Zhang, Nucl. Phys. B 491, 3 (1997) [arXiv:hep-ph/9606211].

[11] J. R. Ellis, M. K. Gaillard, D. V. Nanopoulos and S. Rudaz, Nucl. Phys. B 176, 61 (1980).

[12] Y. Aoki, C. Dawson, J. Noaki and A. Soni, Phys. Rev. D 75, 014507 (2007) [arXiv:hep-lat/0607002].

[13] J. E. Kim, Phys. Rev. Lett. 43, 103 (1979); M. A. Shifman, A. I. Vainshtein and V. I. Zakharov, Nucl. Phys. B 166, 493 (1980).

[14] A. R. Zhitnitsky, Sov. J. Nucl. Phys. 31 (1980) 260 [Yad. Fiz. 31 (1980) 497]; M. Dine, W. Fischler and M. Srednicki, Phys. Lett. B 104, 199 (1981).

[15] C. A. Baker et al., Phys. Rev. Lett. 97, 131801 (2006) [arXiv:hep-ex/0602020].

[16] G. G. Raffelt, Lect. Notes Phys. 741, 51 (2008) [arXiv:hep-ph/0611350].

[17] E. Nardi, F. Sannino and A. Strumia, JCAP 0901, 043 (2009) [arXiv:0811.4153 [hep-ph]]; P. Meade, M. Papucci, A. Strumia and T. Volansky, arXiv:0905.0480 [hep-ph].

[18] A. Ibarra and D. Tran, JCAP 0902, 021 (2009) [arXiv:0811.1555 [hep-ph]]; A. Ibarra, D. Tran and C. Weniger, arXiv:0906.1571 [hep-ph].

[19] S. Shirai, F. Takahashi and T. T. Yanagida, arXiv:0905.0388 [hep-ph].

[20] C. H. Chen, C. Q. Geng and D. V. Zhuridov, arXiv:0905.0652 [hep-ph].

[21] J. L. Feng, T. Moroi, L. Randall, M. Strassler and S. f. Su, Phys. Rev. Lett. 83, 1731 (1999).

[22] See, for example, the result in M. Fujii, M. Ibe and T. Yanagida, Phys. Lett. B 579, 6 (2004) [arXiv:hep-ph/0310142].

[23] J. Hisano, S. Matsumoto, M. Nagai, O. Saito and M. Senami, Phys. Lett. B 646, 34 (2007) [arXiv:hep-ph/0610249].

[24] K. Ishiwata, S. Matsumoto and T. Moroi, JHEP 0905, 110 (2009) [arXiv:0903.0242 [hep-ph]].

[25] W. Buchmuller, A. Ibarra, T. Shindou, F. Takayama and D. Tran, arXiv:0906.1187 [hep-ph].

[26] T. Sjostrand, S. Mrenna and P. Skands, JHEP 0605, 026 (2006) [arXiv:hep-ph/0603175].

[27] A. Ibarra and D. Tran, JCAP 0807, 002 (2008) [arXiv:0804.4596 [astro-ph]].

[28] J. Chang et al., Nature 456, 362 (2008).

[29] S. Torii et al., arXiv:0809.0760 [astro-ph].

[30] F. Aharonian et al. [H.E.S.S. Collaboration], Phys. Rev. Lett. 101, 261104 (2008) [arXiv:0811.3894 [astro-ph]].

[31] E. A. Baltz and J. Edsjo, Phys. Rev. D 59 (1999) 023511 [arXiv:astro-ph/9808243].

[32] M. Aguilar et al. [AMS-01 Collaboration], Phys. Lett. B 646, 145 (2007) [arXiv:astro-ph/0703154].

[33] S. W. Barwick et al. [HEAT Collaboration], Astrophys. J. 482, L191 (1997) [arXiv:astro-ph/9703192].

[34] O. Adriani et al., Phys. Rev. Lett. 102, 051101 (2009) [arXiv:0810.4994 [astro-ph]].

[35] C. R. Chen, M. M. Nojiri, S. C. Park, J. Shu and M. Takeuchi, arXiv:0903.1971 [hep-ph].

[36] J. Hisano, S. Matsumoto, O. Saito and M. Senami, Phys. Rev. D 73, 055004 (2006) [arXiv:hep-ph/0511118].

[37] M. Ibe and T. Watari, Phys. Rev. D 67, 114021 (2003) [arXiv:hep-ph/0303123].

[38] M. Cirelli and A. Strumia, arXiv:0903.3381 [hep-ph].

[39] P. L. Brink et al. [CDMS-II Collaboration], In the Proceedings of 22nd Texas Symposium on Relativistic Astrophysics at Stanford University, Stanford, California, 13-17 Dec 2004, pp 2529 [arXiv:astro-ph/0503583].

[40] M. Ibe, T. Moroi and T. T. Yanagida, Phys. Lett. B 644, 355 (2007) [arXiv:hep-ph/0610277]. 